\title{
GUIA PARA A DETERMINAÇÃo DA ESTABILIDADE DE PRODUTOS QUÍMICOS
}

\author{
Luciana R. Oriqui e Milton Mori* \\ Faculdade de Engenharia Química, Universidade Estadual de Campinas, Av. Albert Einstein, 500, 13083-852 Campinas - SP, Brasil \\ Pedro Wongtschowski \\ Grupo Ultra, São Paulo - SP, Brasil
}

Recebido em 3/4/12; aceito em 28/8/12; publicado na web em 6/2/13

\begin{abstract}
GUIDE FOR DETERMINING THE STABILITY OF CHEMICAL PRODUCTS. Companies worldwide are reviewing their working process to avoid waste, become aligned with environmental management standards and to fulfill specifications defined for national and international regulations. In this context, it is important that Brazilian Chemical companies have a specific stability guide for their products. The main purpose of this work is to present a stability guide for chemical products based on the existing guides of the Pharmaceutical and Cosmetics segments. Furthermore, this work proposes to offer an additional period of shelf life for chemical products, provided they meet certain prerequisites.
\end{abstract}

Keywords: stability guide; chemical products; shelf life.

\section{INTRODUÇÃO}

No mercado interno, as indústrias brasileiras e, entre elas, as químicas, estão sujeitas à Lei No 8.078, de 11/9/1990, Lei dos Direitos do Consumidor, ${ }^{1}$ que regulamenta entre outros, a necessidade do prazo de validade e de informações sobre riscos à saúde e segurança dos consumidores serem informados de forma clara e em língua portuguesa sobre os produtos comercializados.

No mercado externo, mais especificamente no mercado europeu, com o advento do regulamento REACH, Registro, Avaliação, Autorização e Restrição de Substâncias Químicas, é necessário um estudo de estabilidade que possibilite o fornecimento de informações e garantias nele definidas para produtos químicos.

O propósito do teste de estabilidade é fornecer evidências de como a qualidade de um produto varia com o tempo sob influência de uma variedade de fatores ambientais, como temperatura, umidade e luz, e estabelecer um prazo de validade para os produtos além de recomendar condições de armazenamento adequadas. O prazo de validade fica então definido como o tempo, desde sua fabricação, em que um produto mantém suas características de qualidade dentro de padrões estabelecidos por lei e/ou aceitáveis por parte do consumidor.

Como não há um Guia de Estudos de Estabilidade específico para o segmento, as indústrias químicas vêm se baseando nos guias nacionais e internacionais vigentes para o segmento farmacêutico e de cosméticos, especialmente o Q1AR2 e Q1E, ${ }^{2,3}$ do International Conference on Harmonisation of Technical Requirements for Registration of Pharmaceuticals for Human Use, ICH, e o Guia de Estabilidade de Produtos Cosméticos, ${ }^{4}$ da ANVISA, e também na Resolução RE no.1 de 29/7/2005, ${ }^{5}$ que revogou a Resolução RE no. 398 , de 12/11/2004, ${ }^{6}$ ambas da ANVISA.

Devido às peculiaridades características a cada área, é relevante que se tenha um Guia específico para produtos químicos. O Guia proposto apresentará regras gerais já que, assim como os similares já regulamentados, objetiva ampla abrangência. Produtos que não se adéquem a esta generalização deverão ser tratados e analisados de acordo com suas especificidades.

\section{DESCRIÇÃO RESUMIDA DO GUIA DE ESTABILIDADE PARA PRODUTOS QUÍMICOS}

O Guia de Estabilidade para Indústrias Químicas baseia-se em parâmetros indicados pelo International Conference on Harmonisation of Technical Requirements for Registration of Pharmaceuticals for Human Use, ICH, para estudos de estabilidade em países de zona climática IV-B, onde se encontra o Brasil.

Será proposta uma metodologia para definição de prazo de validade para os produtos químicos e a indicação, quando apropriado, de um prazo de validade adicional, que chamaremos de prazo de reteste, cujo estudo para validação deve ser efetuado com antecedência mínima de 1 mês do vencimento do prazo de validade inicialmente proposto.

Como demonstrado por Oriqui et al., ${ }^{7}$ o Brasil realmente se adéqua aos parâmetros climáticos da Zona IV-B e, portanto, os testes de estabilidade propostos para a indústria química serão uma adequação dos já adotados para as indústrias farmacêuticas e de cosméticos brasileiras, que foram indicados em função das características climáticas específicas dessa Zona e utilizarão como referência os Guias Q1A(R2) e Q1E do ICH, ${ }^{2,3}$ o Guia de Estabilidade de Produtos Cosméticos e Resoluções da ANVISA.4-6 O guia de estabilidade para produtos químicos é pioneiro no segmento e inova ao propor a possibilidade de prazo de validade adicional, ou prazo de reteste, para produtos que atenderem às garantias mínimas requeridas, respaldados por resultados de testes de estabilidade, análises químicas e quimiométricas.

Apesar de não haver atualmente resoluções da ANVISA tratando especificamente de estudos de estabilidade para a indústria química, empresas do segmento vêm sendo autuadas pela mesma por estarem reprocessando materiais vencidos ou próximos do vencimento que, segundo a Agência, poderiam trazer danos à saúde e ao meio ambiente. ${ }^{8}$

Por esta razão, o Guia proposto adotará os mesmos limites sugeridos pelos guias do $\mathrm{ICH}$, que foram definidos através de pesquisas feitas pelos participantes do órgão e que tendem a ser mais rígidos dos que seriam necessários efetivamente para a indústria química, justamente pelo segmento farmacêutico ser o benchmark, referência, em estudos de estabilidade.

Esta maior margem de segurança inicial garantirá a segurança nos estudos até que possam ser analisados diversos casos práticos para o segmento químico, que garantam a utilização de outros parâmetros.

*e-mail: mori@feq.unicamp.br 


\section{Princípios gerais}

Testes de estabilidade são experimentos conduzidos em condições pré-estabelecidas de temperatura e umidade, que devem representar um modelo das condições climáticas no ambiente em que os produtos serão transportados e armazenados durante seu prazo de validade.

Segundo Huynh-Ba, ${ }^{9}$ o ambiente para definição do prazo de validade pode ser suficientemente descrito por parâmetros que influenciam a estabilidade, principalmente calor e umidade. Há, entretanto, diversos outros fatores de risco, que podem impactar na estabilidade dos produtos:

- fatores internos de reatividade dos ingredientes ativos, excipientes e materiais de embalagem, bem como as possíveis interações entre esses componentes. Os riscos podem ser reduzidos através dos testes de stress como parte do desenvolvimento do produto;

- fatores relativos à produção, como tamanhos dos lotes, equipamentos, qualidade dos componentes. Os procedimentos padrões para reduzir esses riscos são as validações de processos, incluindo Boas Práticas de Fabricação, Qualificação de Instalações, Qualificação Operacional e aplicação de novas tecnologias indicadas pela Tecnologia de Processos Analíticos;

- fatores externos como calor e umidade, luz, pH, oxigênio. Os riscos são reduzidos por testes de longa duração, acelerados e de stress, para identificar a embalagem adequada, prazo de validade e recomendações de armazenamento;

- danos físicos durante o transporte e armazenamento. Embalagens secundárias adequadas (tambores, caixas e containers) reduzem esse risco.

Pedro $^{10}$ alerta sobre a dificuldade em se definir claramente os parâmetros que definem a qualidade de um produto e seus níveis mínimos aceitáveis, mas ressalta que a qualidade basicamente pode ser dividida em três grupos principais:

- Segurança: os produtos não podem conter nenhum agente não seguro ao consumidor, mesmo após seu uso como, por exemplo, o desenvolvimento de agentes nocivos microbiológicos ou químicos. Impactos ambientais de produção, distribuição, uso e descarte de produtos também devem ser considerados.

- Aspectos primários ou funcionais: existem para que o produto possa cumprir sua função principal e, portanto, devem permanecer eficazes durante todo o prazo de validade do produto.

- Aspectos secundários: apesar de não serem responsáveis por nenhum aspecto funcional do produto, existem geralmente para agregar valor e se tornarem fatores de diferenciação de marcas. Exemplo: embalagens de fácil manuseio e abertura.

\section{Guia de estudos de estabilidade para produtos químicos}

\section{Testes de stress ou testes de esforço}

Os testes de stress fazem parte das estratégias de desenvolvimento de produtos e são normalmente realizadas sob condições mais severas que as dos testes acelerados. Eles ajudam a identificar os prováveis produtos da degradação, caso ela ocorra em condições aceleradas ou de longo prazo de armazenamento.

A identificação destes produtos possibilita o esclarecimento dos caminhos da degradação e a estabilidade intrínseca da molécula, informações estas bastantes relevantes, principalmente para o quesito segurança do produto e, portanto, para especificações do REACH.

Os testes de stress podem ser feitos em um único lote da substância. Os ensaios devem incluir os efeitos:

- da temperatura, com incrementos de $10^{\circ} \mathrm{C}$ (por exemplo, 50, 60 ${ }^{\circ} \mathrm{C}, \ldots$ ) acima da temperatura para testes acelerados;

- da umidade (por exemplo, $80 \%$ de umidade relativa ou mais), com percentual apropriado para ocorrência de oxidação e fotólise dos produtos.

Os ensaios devem também avaliar a suscetibilidade dos produtos à hidrólise em uma ampla faixa de $\mathrm{pH}$, quando em solução ou suspensão.

Os testes de fotoestabilidade são parte integrante dos testes de stress e têm como objetivo determinar se o produto é sensível à luz. A exposição à radiação luminosa pode, além de alterar cor e odor do produto, levar à degradação de ingredientes da formulação.

Estes estudos podem ser feitos através da exposição do produto a uma fonte de iluminação que pode ser a luz solar captada através de vitrines especiais para esse fim ou lâmpadas que apresentam espectro de emissão semelhante ao do Sol como, por exemplo, as lâmpadas de xenônio ou fontes de luz ultravioleta. Os testes devem ser conduzidos com exposição direta do produto à fonte de iluminação. Se não houver alterações inaceitáveis, os testes se encerram neste momento. Caso elas ocorram, são necessários testes adicionais de fotoestabilidade do produto em sua embalagem primária e possivelmente também na secundária.

Embalagem primária é aquela que se encontra em contato direto com o produto químico e embalagem secundária contém as embalagens primárias que chegam até o consumidor. Por exemplo, em um produto embalado em filme laminado e depois em tambor, o filme seria a embalagem primária e o tambor seria a embalagem secundária.

Os resultados destes estudos têm um papel crítico no desenvolvimento da formulação bem como no desenvolvimento de embalagens.

É indicado que estudos de fotoestabilidade sejam realizados em um lote de cada formulação, para verificação de possíveis alterações químicas ou físicas. Se houver diminuição do teor do ingrediente ativo ou aumento dos produtos de degradação, então podem ser necessárias tanto uma nova embalagem que confira maior proteção ao produto, quanto uma etiqueta de alerta e instruções.

\section{Seleção de lotes}

Os lotes dos produtos fornecidos para o estudo formal de estabilidade devem ser homogêneos e com qualidade geral representativa da qualidade do material produzido em escala usual de produção. Devem também ser fabricados a partir de diferentes lotes de componentes ativos. A utilização de um único lote de componente ativo minimizaria a robustez dos resultados e impactaria no cálculo do prazo de validade do produto.

Para fins de estudos de estabilidade em novos produtos, devem ser selecionados um lote em escala piloto e dois outros que podem ser em menor escala, mas ainda assim representativos no que diz respeito às etapas críticas de produção, para comparação e validação de resultados.

Um lote em escala piloto deve ser representativo em relação às etapas críticas de produção do produto em estudo e deve simular o lote de produção industrial em uma quantidade mínima equivalente a $10 \%$ do lote industrial previsto, ou então ser equivalente à capacidade mínima do equipamento industrial a ser utilizado. Deve ainda usar o mesmo método de manufatura e procedimentos que no processo final.

Já para produtos estabelecidos no mercado (que já passaram pela fase inicial onde foram feitos os estudos de estabilidade que definiram os "caminhos da degradação" através de testes de stress e o prazo de validade através de estudos acelerados e de longa duração), para cálculos de possíveis prazos de reteste, devem ser selecionadas pelo menos 30 amostras representativas de cada lote comercializado, acondicionadas segundo critérios indicados em "Material de acondicionamento".

A indicação mínima de 30 amostras possibilita que a distribuição das médias amostrais possa ser aproximada por uma distribuição normal (teorema do limite central), garantia esta necessária para futuros cálculos estatísticos em extrapolações do prazo de reteste. 


\section{Material de acondicionamento}

Os estudos de estabilidade devem ser realizados em produtos embalados em recipientes com sistema de fechamento idêntico, ou ao menos bastante similar, ao das embalagens propostas para armazenamento e distribuição.

De maneira semelhante às substâncias farmacêuticas, quando o produto químico em estado sólido é armazenado em filme laminado contido em tambor de papelão, por exemplo, os estudos de estabilidade são dificultados pela falta de espaço e pela quantidade de produto necessário. Nestas situações, pequenos tambores devem ser utilizados. É relevante que se tome cuidado para que a espessura desse novo cilindro não forneça maior ou menor proteção que o tambor original.

Uma alternativa à embalagem idêntica à de armazenamento e distribuição, é, assim como na indústria de cosméticos, ${ }^{4}$ fazer os testes de estabilidade em duas etapas paralelas:

1. acondicionamento das amostras em frasco de vidro neutro, transparente, com tampa que garanta boa vedação evitando perda de gases ou vapor para o meio. A quantidade de produto para as amostras que passarão pelos testes deve ser suficiente para as avaliações necessárias. Se houver incompatibilidade conhecida entre componentes da formulação e o vidro, deve-se selecionar outro material de acondicionamento, compatível com o produto a ser analisado;

2. avaliação de compatibilidade entre a formulação e o material de acondicionamento final.

Durante o envase no recipiente de teste, deve-se evitar incorporação de ar no produto e também não completar o volume total da embalagem, permitindo um espaço vazio (head space) de aproximadamente um terço da capacidade do frasco para possíveis trocas gasosas. $^{4}$

\section{Especificações}

Estudos de estabilidade devem incluir testes para atributos físicos, químicos e microbiológicos em produtos que são suscetíveis a alterações que influenciam sua qualidade, segurança e/ou eficácia, durante o armazenamento.

Aspectos a serem considerados:

- aspectos físicos: devem ser conservadas as propriedades físicas originais como aspecto, cor, odor, uniformidade, dentre outras;

- aspectos químicos: devem ser mantidos dentro dos limites especificados a integridade da estrutura química, o teor de ingrediente ativo e outros parâmetros pré-definidos pelo fabricante do produto;

- aspectos microbiológicos: devem ser conservadas as características microbiológicas, conforme os requisitos especificados. O cumprimento das Boas Práticas de Fabricação e os sistemas conservantes utilizados na formulação podem garantir estas características.

Também é relevante que seja garantida a manutenção das características do produto em relação à:

- funcionalidade: os atributos do produto devem ser mantidos sem alterações quanto ao efeito inicial proposto;

- segurança: não devem ocorrer alterações significativas que influenciem na segurança de uso do produto. Os níveis de impureza devem ser controlados para a definição eficaz do prazo de validade.

\section{Frequência dos testes}

É preciso estabelecer, através de estudos de longo prazo, o perfil de estabilidade para o produto durante, no mínimo, todo o prazo de validade a ser proposto.

Nestes estudos de longo prazo, a frequência dos testes para proposição de prazo de validade de pelo menos 12 meses, deve ser: a cada 3 meses no primeiro ano de validade; a cada 6 meses no segundo ano e, anualmente no restante do período proposto, se este for maior que 24 meses.

Para adoção de um prazo provisório, que possibilite a entrada de um produto no mercado antes de aguardar o resultado completo dos estudos de longa duração pelo prazo integral proposto, são realizados estudos de estabilidade em condições aceleradas de armazenamento.

Para um prazo provisório de 24 meses, usualmente adotado no mercado, a frequência dos testes de estudos em condições aceleradas deve ser um estudo de 6 meses, onde é recomendado um mínimo de 3 pontos na escala de tempo, incluindo a formação inicial e momentos finais ( 0,3 e 6 meses).

Quando existirem expectativas (baseadas na experiência de desenvolvimento) e/ou houver confirmação que os resultados de estudos acelerados sejam suscetíveis de mudanças significativas nas especificações do produto, é importante incrementar os estudos, incluindo testes em condições intermediárias.

Nestes casos, onde são requeridos testes em condições intermediárias de armazenamento, o período de estudos é de 12 meses, e é recomendado um mínimo de 4 pontos nas escala do tempo, incluindo o início e o fim $(0,6,9$ e 12 meses $)$.

\section{Condições de armazenamento}

Em geral, os produtos devem ser avaliados em condições reais de armazenamento (com tolerâncias apropriadas) que testem sua estabilidade térmica e, se aplicável, sua sensibilidade à umidade. As condições de armazenamento e o prazo para os estudos escolhidos devem ser suficientes para cobrir o armazenamento, expedição e posterior utilização do produto.

Dados de condições aceleradas de armazenamento e, se apropriado, de condições intermediárias de armazenamento, são utilizados para avaliar o efeito de exposições de curto prazo fora das condições ideais de armazenamento, como, por exemplo, pode ocorrer durante o transporte do produto.

Serão consideradas alterações significativas, a incapacidade do produto em satisfazer suas especificações, nestas condições:

- redução superior a 5\% do teor do componente ativo inicial;

- qualquer produto de degradação especificado que exceda o critério de aceitação;

- não cumprimento dos critérios de aceitação para aparência e propriedades físicas (por exemplo, cor, separação de fases, dureza, aglomerantes, entre outros) apropriadas a cada produto;

- pHs diferentes de seus critérios de aceitação.

Serão entendidos como produtos de degradação, produtos que se formem por polimerização, condensação, oxidação ou quaisquer outros processos que resultem em alterações nas especificações iniciais.

Usualmente, produtos de degradação que possuem poucas informações sobre sua segurança devem ser limitados em níveis que fiquem entre 0,2 e $0,5 \%$ do teor do componente ativo no final do prazo de validade proposto. ${ }^{9}$

É recomendado um intervalo pré-definido para as condições de temperatura e umidade relativa nas câmaras de armazenamento: temperaturas devem ser controladas com margem de erro de $2{ }^{\circ} \mathrm{C}$ $\left( \pm 2{ }^{\circ} \mathrm{C}\right)$ e umidades relativas com margem de erro de $5 \%( \pm 5 \%)$.

As condições de armazenamento básicas a serem testadas em estudos de estabilidade são: estocagem em temperatura elevada, ciclos de temperatura (ambiente, elevada e baixa), atmosferas com variação de umidade relativa, exposição à luz e transições de fases (ciclos de congelamento e descongelamento, por exemplo).

Os testes de exposição à radiação luminosa verificam alterações da cor e odor do produto e, também, alterações nas especificações iniciais de ingredientes da formulação.

Em relação aos testes de temperatura, os de longo prazo devem ter 
uma duração mínima de 12 meses, desde sua apresentação e por um período de tempo suficiente para cobrir o prazo de validade proposto, e devem ser feitos pelo menos nos 3 lotes indicados para os estudos formais de estabilidade.

Para adoção de um prazo de validade provisório de 24 meses, assim como na indústria farmacêutica onde o procedimento já é regulamentado, ${ }^{5}$ deve-se garantir que:

- tenham sido feitos estudos de estabilidade acelerada de 6 meses, acrescidos de resultados preliminares de estudos de longa duração pelo mesmo período;

- a substância ativa seja considerada estável, ou seja, não facilmente degradável;

- nenhuma mudança significativa tenha sido observada nos estudos de estabilidade acelerada;

- dados de apoio demonstrem similaridade com formulações para as quais tenham sido atribuídos e confirmados prazos de validade iguais ou superiores a 24 meses;

- será dada continuidade aos estudos de longa duração até cobrir o prazo de validade proposto.

O prazo de validade de um produto químico a ser comercializado no Brasil deverá ser determinado por um estudo de estabilidade de longa duração, de acordo com os parâmetros definidos na Tabela 1 (parâmetros para a Região IV-B, onde se encontra o Brasil).

As especificações mínimas de qualidade são características de cada produto e devem ser definidas pelos fabricantes. Nas condições de testes de estabilidade indicadas na Tabela 1, devem ser feitas análises (análises térmicas, espectrofotométricas e cromatográficas, por exemplo) cujas técnicas sejam sensíveis às informações a serem monitoradas.

\section{Prazo de validade $x$ mudanças significativas}

Adotando para produtos químicos o mesmo critério adotado pelo $\mathrm{ICH}$, no Guia Q1A(R2), ${ }^{2}$ segue:

$\checkmark$ se mudanças significativas ocorrerem entre 3 e 6 meses, em condições de armazenamento acelerado: o prazo de validade proposto deve ser baseado no tempo real disponibilizado pelo teste de armazenamento a longo prazo. Caso não haja mudanças significativas nesse período e se devidamente acompanhada de resultados preliminares de estudo de longa duração, pode ser atribuído prazo provisório de 24 meses;

$\checkmark$ se mudanças significativas ocorrem antes dos 3 primeiros meses em condições de armazenamento acelerado: deve-se fazer uma análise sobre os possíveis efeitos da exposição do produto por períodos de curto prazo fora das condições recomendadas de armazenamento (por exemplo, durante o transporte e manuseio). A discussão pode ser apoiada, se for o caso, por mais testes em um único lote de produto, em um período menor que 3 meses, mas com maior frequência nos testes que o usual. É considerado desnecessário continuar a testar o produto por 6 meses, quando uma mudança significativa ocorreu antes dos 3 primeiros meses.

Para estudar os efeitos da exposição dos produtos por curtos períodos fora das condições recomendadas de armazenamento, recomenda-se que, além dos estudos de estabilidade, sejam feitos testes de transporte e distribuição. Estes estudos têm a finalidade de antecipar o comportamento do produto em sua cadeia logística, incluindo manuseio e transporte.

Testes de transporte (simulações que envolvem vibração, pressão, teste de queda - drop test - e variações ambientais - umidade e temperatura) são indicados para simular condições agravantes durante $o$ transporte, que podem interferir, entre outros, na aparência do produto e na funcionalidade da embalagem.

\section{Prazo de validade e extrapolações}

Na definição do prazo de validade de um produto, independentemente de qual parâmetro esteja sendo estudado, é usual que ele sofra certas alterações com o decorrer do tempo.

Determinar o prazo de validade pode muitas vezes ser a etapa mais lenta para o lançamento de um produto no mercado. Isto é particularmente importante caso haja problemas na estabilidade que requeiram alterações na formulação ou no processo.

Em princípio, o prazo de validade pode ser determinado através da realização de testes adequados e em tempo real, longa duração, até que os limites de preservação dos parâmetros pré-estabelecidos para os produtos sejam atingidos. Na prática, pesquisadores e empresas, em razão de recursos e tempo, querem uma indicação da estabilidade sem esperar por todo o prazo de validade previsto, que usualmente é maior ou igual a 24 meses.

As empresas usualmente determinam o prazo de validade sob condições aceleradas para assegurarem que processo e formulação não gerarão problemas de estabilidade em um estágio adiantado do desenvolvimento de produtos. No lançamento de novos produtos, o prazo de validade muitas vezes é atribuído com base em dados que envolvem certa extrapolação dos dados acelerados, ao invés de se estenderem em estudos por todo o período de prazo de validade indicado.

Tabela 1. Testes de estabilidade para a região IV-B

\begin{tabular}{|c|c|c|c|c|}
\hline Produto & $\begin{array}{c}\text { Condição de } \\
\text { armazenamento a ser } \\
\text { informada no rótulo* }\end{array}$ & Embalagem & Testes em condições aceleradas** & Teste de longa duração** \\
\hline Sólido & $15-30^{\circ} \mathrm{C}$ & Semipermeável & $40 \pm 2^{\circ} \mathrm{C} / 75 \pm 5 \% \mathrm{UR}$ & $30 \pm 2{ }^{\circ} \mathrm{C} / 75 \pm 5 \% \mathrm{UR}$ \\
\hline $\begin{array}{c}\text { Sólido } \\
\text { Semissólido }^{* * *}\end{array}$ & $\begin{array}{l}15-30^{\circ} \mathrm{C} \\
15-30^{\circ} \mathrm{C}\end{array}$ & $\begin{array}{l}\text { Impermeável } \\
\text { Semipermeável }\end{array}$ & $\begin{array}{c}40 \pm 2{ }^{\circ} \mathrm{C} \\
40 \pm 2^{\circ} \mathrm{C} / 75 \pm 5 \% \text { UR }\end{array}$ & $\begin{array}{c}30 \pm 2{ }^{\circ} \mathrm{C} \\
30 \pm 2^{\circ} \mathrm{C} / 75 \pm 5 \% \text { UR }\end{array}$ \\
\hline $\begin{array}{l}\text { Semissólido } \\
\text { Líquido*** }\end{array}$ & $\begin{array}{l}15-30^{\circ} \mathrm{C} \\
15-30^{\circ} \mathrm{C}\end{array}$ & $\begin{array}{l}\text { Impermeável } \\
\text { Semipermeável }\end{array}$ & $\begin{array}{c}40 \pm 2^{\circ} \mathrm{C} \\
40 \pm 2^{\circ} \mathrm{C} / 75 \pm 5 \% \text { UR }\end{array}$ & $\begin{array}{c}30 \pm 2{ }^{\circ} \mathrm{C} \\
30 \pm 2^{\circ} \mathrm{C} / 75 \pm 5 \% \text { UR }\end{array}$ \\
\hline $\begin{array}{c}\text { Líquidos } \\
\text { Gases } \\
\text { Todos estados }\end{array}$ & $\begin{array}{c}15-30^{\circ} \mathrm{C} \\
15-30^{\circ} \mathrm{C} \\
2-8^{\circ} \mathrm{C}\end{array}$ & $\begin{array}{l}\text { Impermeável } \\
\text { Impermeável } \\
\text { Impermeável }\end{array}$ & $\begin{array}{l}40 \pm 2^{\circ} \mathrm{C} \\
40 \pm 2^{\circ} \mathrm{C} \\
25 \pm 2^{\circ} \mathrm{C}\end{array}$ & $\begin{array}{c}30 \pm 2^{\circ} \mathrm{C} \\
30 \pm 2^{\circ} \mathrm{C} \\
5 \pm 3{ }^{\circ} \mathrm{C}\end{array}$ \\
\hline Todos estados & $2-8^{\circ} \mathrm{C}$ & Semipermeável & $25 \pm 2{ }^{\circ} \mathrm{C} / 60 \pm 5 \% \mathrm{UR}$ & $5 \pm 3{ }^{\circ} \mathrm{C}$ \\
\hline Todos estados & $-20^{\circ} \mathrm{C}$ & Todas & $-20 \pm 5^{\circ} \mathrm{C}$ & $-20 \pm 5^{\circ} \mathrm{C}$ \\
\hline
\end{tabular}

*qualquer recomendação de armazenamento em temperaturas dentro dessas faixas deve constar nas embalagens do produto; **os valores de temperatura e umidade dos testes de estabilidade são fixos e as variações são devidas às oscilações esperadas na câmara climática e aberturas possíveis para a remoção ou a entrada de material; ***os estudos de líquidos à base de água e produtos semissólidos devem ser conduzidos a 25 ou $75 \%$ de umidade relativa (UR). Caso se opte por $75 \%$ de UR, o valor da perda de peso deve ser multiplicado por 3,0. 
Para se fazer uma extrapolação com o tempo, a forma funcional da instabilidade torna-se importante. Em processos químicos, a formação de produtos de degradação ou a perda do teor do ativo inicial envolve um processo cinético.

Para que ocorra uma reação química e produtos de degradação sejam originados, é preciso que haja uma energia local mínima que possibilite colisões entre reagentes. Essa energia mínima necessária é conhecida como energia de ativação, $\mathrm{E}_{\mathrm{a}}$.

Quando a temperatura de um sistema é aumentada, o correspondente incremento de energia (cinética, vibracional, interna) em moléculas resulta no aumento das taxas de reações. Isto leva à relação conhecida como Equação de Arrhenius, que relaciona a taxa de reação $k$ e a temperatura $\mathrm{T}$ (em Kelvin).

$\ln k=\ln \mathrm{A}-\mathrm{E}_{\mathrm{a}} /(\mathrm{RT})$

(Equação de Arrhenius)

onde, $k=$ taxa de reação; $\mathrm{T}=$ temperatura (em Kelvin); $\mathrm{A}=$ termo de proporcionalidade (também conhecido como "Fator-A"); $\mathrm{R}=$ constante gasosa $\left(1,987 \mathrm{cal} \mathrm{K}^{-1} \mathrm{~mol}^{-1}\right.$ ou $\left.8,314 \mathrm{~J} \mathrm{~K}^{-1} \mathrm{~mol}^{-1}\right)$

Estudos indicam que, tipicamente, reações químicas têm energia de ativação entre 10 e 30 kcal/mol e Gallardo, Rojas e Flórez indicam o uso de $20 \mathrm{kcal} / \mathrm{mol}$ como um valor referência de energia de ativação, já que este foi o resultado de uma revisão de 132 publicações entre 1950 e $1980 .{ }^{11}$

A energia de ativação também pode ser determinada experimentalmente através do cálculo da inclinação do gráfico de Arrhenius, que por sua vez pode ser usado para predizer a taxa de formação de produtos de degradação (ou perda do teor de ativo inicial) em condições de armazenamento.

O gráfico de Arrhenius (isto é, ln $k$ versus 1/T) pode ser construído uma vez que os estudos de estabilidade em condições aceleradas são conduzidos a elevadas temperaturas.

O prazo de validade corresponderá ao tempo necessário para atingir os níveis limítrofes de degradantes (ou de perda do teor de ativo inicial)

Huynh- $\mathrm{Ba}^{9}$ indica para cálculo desse tempo, a Equação para proposição de prazo de validade a seguir:

$$
\text { Prazo de Validade }=\left([\mathrm{D}]-\left[\mathrm{D}_{0}\right]\right) / \mathrm{A} \exp \left(\mathrm{E}_{\mathrm{a}} / \mathrm{RT}\right)
$$

onde: $\mathrm{T}$ = temperatura absoluta para as condições de armazenamento; [D] = concentração limite de produtos de degradação permitida para o prazo de validade; $\left[\mathrm{D}_{0}\right]=$ concentração de produtos de degradação presentes inicialmente.

Interpolações de estabilidade assumem que o mesmo comportamento dos dados obtidos, linear ou curvo, continuará além do período coberto por estudos de longo prazo. Mesmo que uma aproximação linear seja válida, a incerteza deve estar refletida no intervalo de confiança dos dados medidos.

Métodos estatísticos devem ser aplicados para verificação do ajuste adequado da regressão linear ou curva assumida para os dados de longo prazo. Em alguns softwares, esse ajuste, ou a falta dele pode ser obtido pela informação lack of fit.

No uso de extrapolações em estudos de estabilidade para estabelecimento de prazos de validade, a precisão na determinação do prazo é afetada tanto pelo montante da extrapolação pretendida, quanto pela própria precisão na coleta de dados.

O tamanho e a preparação da amostra são fatores de influência impactante na coleta de dados, daí a indicação de no mínimo 3 lotes para estudos de estabilidade, todos eles representativos das etapas críticas do processo produtivo.

Vale ressaltar que a precisão das mensurações de estabilidade também é afetada pela procedência das informações: dados provenientes da formação de produtos de degradação ou provenientes da perda do componente ativo. Kenneth C Waterman (apud Huynh-Ba) ${ }^{9}$ demonstra que geralmente a precisão é maior quando são utilizados dados provenientes da formação de produtos de degradação.

Uma abordagem para a análise dos dados quantitativos sobre um atributo que se espera que venha a se alterar com o tempo, é a de trabalhar com $95 \%$ de confiabilidade e determinar o momento em que o limite de confiança bilateral para a curva da média intercepta o critério de aceitação.

Em geral, atributos quantitativos como, por exemplo, produtos de degradação e conteúdo conservante, seguem uma cinética de ordem zero em armazenamento de longo prazo e, portanto, são aptos a análises estatísticas obtidas por regressão linear (ICH, Guia Q1E). ${ }^{3}$

\section{Prazo de reteste e extrapolações}

Quando se fizer necessário, e amparados por estudos de estabilidade, é possível que fabricantes de produtos químicos possam fazer uso da atribuição de prazo de validade complementar ao inicialmente estabelecido, extrapolando dados de estudos de longa duração específicos do lote em estudo e fazendo uso dos dados de estudos acelerados e/ou intermediários iniciais. A esse novo prazo, atribuir-se-á a denominação "prazo de reteste".

Para que seja requerido o prazo de validade adicional, ou prazo de reteste, os estudos para extrapolação devem ter início no mínino 1 mês antes do término do prazo de validade original. Anteceder-se 1 mês à data final de validade, evita que o produto chegue a vencer no estoque das empresas e gere possibilidade de autuação, além de propiciar tempo mínimo hábil para os testes analíticos que se fizerem necessários e que podem vir a propiciar prazo de reteste, caso seja averiguado que não houve alterações significativas no produto até a data de análise para reteste.

As análises a serem feitas no produto devem apurar os seguintes padrões, para confirmação da ausência de alterações significativas:

I) a perda do teor do componente ativo deve ser igual ou inferior a $3 \%$ do valor inicial (margem de segurança de $2 \%$ em relação ao limite usual de $5 \%$ );

II) ausência de produtos de degradação que excedam seus critérios de aceitação;

III) propriedades físicas (aspecto, cor, odor, entre outras), químicas (integridade da estrutura química, $\mathrm{pH}$ adequado, por exemplo) e microbiológicas mantidas de acordo com especificações e tolerâncias de cada produto.

O prazo de validade adicional, ou prazo de reteste, poderá ser aplicável ao produto, ou em outros produzidos sob circunstâncias similares, desde que:

- tenham sido resguardadas as condições mínimas de armazenamento estabelecidas para a boa conservação do produto;

- que estejam disponíveis os dados de estudos acelerados e/ou intermediários daquela formulação e dados de longa duração para o lote específico em análise;

- os testes analíticos confirmem os padrões mínimos das especificações estabelecidas para viabilizar o prazo de reteste.

Como o prazo de reteste será cabível somente quando os dados dos estudos de estabilidade de longa duração (específico do lote) e acelerados (obtidos para proposição do prazo de validade informado para o produto) apresentarem baixa degradação e variabilidade em relação aos dados originais (limites máximos estabelecidos de presença de produtos de degradação e de perda da potência do teor ativo), assume-se que o padrão de alterações durante esse novo prazo a ser proposto será o mesmo obtido nos estudos de estabilidade de longa duração.

A extensão da extrapolação dependerá dos dados de longa duração para os atributos serem passíveis ou não de análises estatísticas. 
O prazo de reteste extrapolado é apropriado dependendo do grau de conhecimento sobre os padrões de mudanças, principalmente do "caminho de formação de produtos de degradação", do bom ajuste de qualquer modelo matemático e da existência de dados de apoio relevantes, ou seja, dados de longo prazo satisfatórios relativos a amostras que tenham sido formuladas e acondicionadas em embalagens bastante semelhantes às de comercialização do produto.

Ao estimar uma regressão linear ou curva que se ajuste aos dados de longo prazo, os próprios dados possibilitam uma verificação do padrão de alterações assumido e ferramentas estatísticas podem ser aplicadas para testar a qualidade do ajuste dos dados à reta ou à curva estimada. Em extrapolações, caso do prazo de reteste, essa verificação interna não é possível, portanto, se faz necessário validar essas informações, assim que possível, com dados obtidos em novo estudo de longo prazo durante esse período extra.

Esses novos dados de longo prazo deverão ser utilizados tanto para ratificar as considerações feitas para as extrapolações do prazo de reteste, quanto para novos dados de entrada caso se considere a proposição de um segundo prazo para o produto em estudo, garantidas as mesmas condições a respeito de alterações significativas, que as estabelecidas para indicação do primeiro prazo.

A natureza de qualquer relação de degradação irá determinar se os dados devem ser transformados para análise da regressão linear. Geralmente a relação pode ser representada por uma função linear, quadrática ou cúbica numa escala aritmética ou logarítmica. Métodos estatísticos devem ser utilizados para testar o bom ajuste dos dados de todos os lotes e de lotes combinados (quando necessário) em relação à linha ou curva de degradação assumida. ${ }^{3}$

O Guia Q1E, ${ }^{3}$ do ICH, especifica quanto e quando extrapolações podem ser consideradas para proposição de período de reteste para substâncias farmacêuticas e prazo de validade para medicamentos, que se estendam além dos dados provenientes de estudos de longa duração. Os estudos e considerações feitos serão utilizados como parâmetros para proposição do prazo de reteste em produtos químicos.

Os dados de estabilidade de longa duração para extrapolações de prazo de reteste devem ser provenientes no mínimo de 30 amostras, embaladas de forma semelhante ao produto posto no mercado, selecionadas aleatoriamente no lote específico para proposição do novo prazo.

Apesar da seleção criteriosa dos lotes iniciais para cálculo do prazo de validade, o grau de variabilidade dos lotes individuais afeta a confiabilidade de que futuros lotes de produção permanecerão dentro dos critérios de aceitação na extrapolação proposta para o prazo de reteste, portanto, são necessários estudos de estabilidade de longa duração específicos para lotes de produtos que se tenha a intenção de proposição de prazos adicionais.

Informações de estabilidade devem incluir, quando apropriado, resultados de testes físicos, químicos, biológicos e microbiológicos. Balanços de massas devem ser avaliados e fatores que possam afetar esses balanços devem ser considerados como, por exemplo, os mecanismos de degradação, a capacidade de leitura dos indicadores de estabilidade e as variações inerentes de procedimentos analíticos. ${ }^{3}$

Como um produto químico pode ter um ou vários atributos críticos de conformidade, cada um deles deve ser avaliado individualmente e o prazo a ser proposto não deve exceder o mais crítico, isto é, o menor previsto entre eles.

O Fluxograma $1 \mathrm{~S}$, material suplementar, é uma adaptação do Apêndice A do Guia Q1E, ${ }^{3} \mathrm{ICH}$, e apresenta um passo-a-passo para avaliação de dados de estabilidade e quando e quanto de extrapolação pode ser considerada para a proposição de prazo de reteste (Tabela 2).

\section{Análises estatísticas}

Ferramentas e métodos estatísticos são amplamente utilizados
Tabela 2. Legenda para Fluxograma 1 - extrapolação de dados de longo prazo para proposição de prazo de reteste

Significado

1 Tabular e/ou plotar os dados de estabilidade em todos os atributos de qualidade e em todas as condições de armazenamento e avaliar cada atributo separadamente

2 Houve alterações significativas em condições aceleradas durante o prazo de 6 meses?

3 Sem extrapolação: não há possibilidade de prazo de reteste

4 Os dados de longo prazo mostram: (a) pouca ou nenhuma alteração ao longo do tempo e (b) pouca ou nenhuma variabilidade?

5 Os dados em condições aceleradas mostram: (a) pouca ou nenhuma alteração ao longo do tempo e (b) pouca ou nenhuma variabilidade?

6 Análises estatísticas são normalmente desnecessárias

$7 \quad Y_{\text {máx }}=2 X$, mas não superior a $X+12$ meses; ou se refrigerado, $\mathrm{Y}_{\max }=1,5 \mathrm{X}$, mas não superior a $\mathrm{X}+6$ meses

8 (a) Dados de longo prazo passíveis de análise estatística e (b) análise estatística realizada?

9 Se apoiado por análises estatísticas e dados relevantes de apoio, então $Y_{\text {máx }}=2 X$, mas não superior a $X+12$ meses; ou se refrigerado, $\mathrm{Y}_{\text {máx }}=1,5 \mathrm{X}$, mas não superior a $\mathrm{X}+6$ meses

10 Se apoiado por dados relevantes de apoio: $\mathrm{Y}_{\text {max }}=1,5 \mathrm{X}$, mas não superior a $X+6$ meses; ou se refrigerado, $Y_{\text {máx }}=X+3$ meses*exposições de curto prazo: exposições fora das condições indicadas de armazenamento (por exemplo, transporte e manuseio); **dados relevantes de apoio: dados de longo prazo satisfatórios no que diz respeito à amostragem e que devem ter sido formulados e acondicionados em embalagens bastante semelhantes às de comercialização do produto

no mercado para garantir confiabilidade e robustez às conclusões de diversos tipos de estudos, principalmente em inferências de dados amostrais e em planejamento de experimentos, entre outros.

Em estudos de estabilidade, a análise de dados de longo prazo a partir de uma confiabilidade estabelecida, usualmente $95 \%$, é utilizada para estimar prazo de validade e intervalos de confiança para variáveis quantitativas, que podem ser atribuído a futuros lotes de produção, desde que os produtos sejam embalados e armazenados em condições similares.

Ferramentas estatísticas disponíveis mais utilizadas na análise e interpretação dos dados de estudo de estabilidade:

$\checkmark$ Análise de regressão: informa como um atributo quantitativo varia de forma sistemática em relação a outra(s) variável(is). A Equação de Arrhenius, por exemplo, é uma equação de regressão linear e o gráfico de Arrhenius traça a relação entre $\ln k$ e 1/T com (-E/R) como a inclinação da reta e $\ln \mathrm{A}$ como intercepto da mesma. Análises de regressão são utilizadas para, através de dados de estabilidade de variáveis quantitativas, especialmente taxa de formação de produtos de degradação, estabelecerem prazos de validade. Extrapolações para períodos superiores aos informados por dados de longo prazo só podem ser feitas se não houverem sido constatadas alterações significativas em condições aceleradas. Além disso, as extrapolações serão apropriadas dependendo do quanto se conhece a respeito do padrão de alterações (já que se assume que esse mesmo padrão continuará pelo período extrapolado além dos dados de longo prazo), do ajuste adequado do modelo matemático e da existência de dados relevantes de apoio.

$\checkmark$ Intervalos de confiança: definem o alcance dos valores para a média do prazo de validade. O intervalo de confiança é dependente da confiabilidade com que se pretende trabalhar. O padrão 
usual é trabalhar com $95 \%$ de confiabilidade. Os limites bilaterais (inferior e superior) são utilizados para comparação com o critério de aceitação do produto. Por exemplo, produtos de degradação costumam aumentar com o tempo, portanto, o limite superior deve ser comparado com o critério de aceitação (o prazo de validade será o menor prazo em que o limite superior cruza o critério de aceitação proposto). Já para teor do componente ativo na formulação, que costuma decrescer com o tempo, o limite inferior deve ser comparado com o critério de aceitação.

$\checkmark$ Amostragem: o correto planejamento dos estudos de estabilidade como, por exemplo, seleção de lotes representativos das etapas críticas de produção e número de amostras, tem influência direta na precisão da inferência e, portanto, na determinação do prazo de validade.

$\checkmark$ Análise de covariância (ANCOVA): utilizada para analisar conjuntamente diversas propriedades, por exemplo, diferentes lotes de produção. Como um estudo preliminar à definição do prazo de validade, a análise de covariância testa as diferentes inclinações e interceptos das regressões entre os diferentes lotes, no intuito de determinar em que momento as diferentes regressões têm inclinação e intercepto comuns. Níveis de confiança de $25 \%$ devem ser utilizados para compensar o baixo poder usual dos testes, decorrente do limitado número de amostras característico de estudos de estabilidade. Basicamente, o objetivo da análise de covariância é testar diferenças entre inclinações e interceptos de regressões lineares entre fatores e combinações de fatores, com o propósito de determinar quando dados de múltiplos fatores podem ser combinados para determinação de um único prazo de validade.

$\checkmark$ Teste de hipóteses: a inferência estatística usa os dados amostrais, essencialmente para estimar um parâmetro populacional e para testar uma afirmativa sobre algum(ns) destes parâmetros populacionais. Essa afirmativa é chamada hipótese. Em função da afirmativa a ser testada, são formuladas as hipóteses nula e alternativa e, a uma dada confiabilidade, são identificados os níveis críticos de aceitação ou não da hipótese nula. Testes de hipótese são utilizados para avaliar, por exemplo, se dados de 2 lotes diferentes têm diferenças significativas.

$\checkmark$ Planejamento de experimentos: estuda o efeito dos fatores (atributos quantitativos e/ou qualitativos) e suas interações. Esse método é bastante eficiente já que possibilita que sejam tiradas conclusões sobre como os fatores interagem para afetar a resposta. Sem o planejamento de experimentos adequado, o efeito dessas interações frequentemente não é levado em consideração, pois ele não é observado. A Quimiometria, por exemplo, pode ser entendida como o uso de um conjunto de métodos estatísticos para o estudo de processos químicos, objetivando o delineamento e análise de experimentos laboratoriais através do planejamento e seleção de condições ótimas de medidas e experimentos para extração do máximo de informação dos dados e, entre os métodos utilizados, ressalta-se o planejamento de experimentos fatoriais.

$\checkmark$ Teorema de Bayes: frequentemente utilizado em análise de decisões, probabilidades denominadas posteriores são calculadas incorporando informações de probabilidades prévias e de probabilidades condicionais.

\section{Declarações/rotulagem}

Uma declaração de armazenagem deve ser estabelecida para a rotulagem, de acordo com os requisitos relevantes nacionais ou regionais. A declaração deve ser baseada na avaliação de estabilidade dos produtos. Quando aplicável, instruções específicas devem ser apresentadas.

\section{CONCLUSÃO}

Como as indústrias químicas vêm, em geral, acompanhando as legislações dos segmentos farmacêuticos e de cosméticos para definirem o prazo de validade de seus produtos, é usual a aplicação do prazo de 24 meses, que é o prazo provisoriamente conferido aos produtos desde que sejam feitos os estudos de estabilidade característicos da região climática onde se encontra o Brasil, VI-B, e que sejam atendidas as seguintes condições: o componente ativo seja considerado estável e nenhuma alteração significativa tenha sido observada; formulações similares apresentem um prazo de validade de 24 meses ou mais; o fabricante dará continuidade ao estudo de longa duração até atingir o prazo de validade proposto e os resultados obtidos deverão ser apresentados imediatamente após a conclusão do estudo.

O problema advindo dessa adoção de um, pode-se dizer, padrão de prazo de validade, é que muitos produtos podem ter validade superior aos 24 meses, mas as indústrias não se sentem confortáveis na extensão da garantia sem a oportunidade de reavaliação do produto terminado este período, para conferência de suas características após armazenamento adequado ou não por parte do consumidor.

Com um guia de estabilidade específico para o setor e a oportunidade de prorrogar o prazo de validade para produtos que mantiverem as especificações mínimas garantidas após o prazo inicialmente proposto, bem como amparadas por extrapolações e análises estatísticas consistentes, as indústrias químicas devem, além de minimizar o impacto ambiental decorrente dos rejeitos, evitar o atual desperdício cujo alto custo tem impactado de forma relevante, tanto o mercado, quanto as próprias empresas.

Os estudos de estabilidade propostos no Guia também deverão se tornar uma fonte de referências e informações de segurança sobre produtos químicos, que possibilitem suporte às indústrias químicas brasileiras para atendimento das exigências do REACH, regulamento europeu concebido para ser uma abordagem integrada a respeito do controle de fabricação, importação e uso de substâncias químicas na Europa.

A partir das diretrizes iniciais indicadas neste trabalho, a próxima etapa consiste na aplicação prática dos conceitos e parâmetros indicados, para obtenção de uma base de dados representativa que possibilite proposição específica de novos limites de extrapolações para prazos de reteste para produtos químicos.

A utilização da Quimiometria garantirá maior acuracidade no entendimento dos caminhos da degradação pela seleção de variáveis importantes em determinado sistema, das alterações de morfologia e de processos de cristalização, entre outros, que por sua vez contribuirá com maior suporte para extrapolações de prazos de reteste e para fornecimento de dados de estabilidade que atendam às demandas do REACH em relação a estudos de estabilidade.

Para a compreensão dos mecanismos de degradação e determinação de prazo de validade de produtos mais complexos, onde o monitoramento de várias características de qualidade se faz necessário, deverá ser considerado o Método Multivariado Acelerado, que une quimiometria e cinética química. ${ }^{10}$

\section{MATERIAL SUPLEMENTAR}

O Fluxograma 1S está disponível em http://quimicanova.sbq.org. br, em arquivo pdf e com acesso livre.

\section{REFERÊNCIAS}

1. Brasil, Lei No 8.078, de 11 de Setembro de 1990; Código de Defesa do Consumidor, disponível em http://www.anvisa.gov.br/legis/consolidada/ lei_8078_90.pdf, acessada em Janeiro 2013. 
2. International Conference on Harmonization - ICH; Q1AR2, Guidance for Industry - Stability Testing of New Drug Substances and Products, 2003, Revision 2, November.

3. International Conference on Harmonization - ICH; Q1E, Evaluation for Stability Data, 2003, Current Step 4 Version, February.

4. Brasil, Agência Nacional de Vigilância Sanitária; Guia de Estabilidade de Produtos Cosméticos, 2004, vol. 1, Maio, disponível em http://www. anvisa.gov.br/divulga/public/series/cosmeticos.pdf, acessada em Janeiro 2013.

5. Brasil, Agência Nacional de Vigilância Sanitária; Resolução RE no. 1, de 29 de julho de 2005, Diário Oficial da União, de 01/08/2005.

6. Brasil, Agência Nacional de Vigilância Sanitária; Resolução RE no. 398, de 12 de novembro de 2004, Diário Oficial da União, de 16/11/2004.
7. Oriqui, L. R.; Mori, M.; Wongtschowski, P.; Freitas, S. R.; Santos, J. G. M.; Quim. Nova 2011, 34, 1869.

8. Formenti, L.; Jornal O Estado de São Paulo, disponível em http://www. estadao.com.br/noticias/impresso, anvisa-autua-duas-empresas-por-vender-agrotoxico-vencido,759540,0.htm, acessada em Janeiro 2013.

9. Huynh-Ba, K. Em Handbook of Stability Testing in Pharmaceutical Development - Regulations, Methodologies and Best Practices; Huynh-Ba, K., ed.; Springer: New York, 2008, chaps. 2-3.

10. Pedro, A. M. K.; Tese de Doutorado, Universidade Estadual de Campinas, Brasil, 2009.

11. Gallardo, C. C.; Rojas, J. J.; Flórez, O. A. A.: Vitae, Revista de La Facultad de Química Farmacêutica 2004, 11, 67. 


\section{GUIA PARA A DETERMINAÇÃo DA ESTABILIDADE DE PRODUTOS QUÍMICOS}

Luciana R. Oriqui e Milton Mori*

Faculdade de Engenharia Química, Universidade Estadual de Campinas, Av. Albert Einstein, 500, 13083-852 Campinas - SP, Brasil Pedro Wongtschowski

Grupo Ultra, São Paulo - SP, Brasil

Considere:

$Y=\underline{\text { Prazo de reteste }}$

$\mathrm{X}=$ período abrangido por dados de longo prazo específicos para o loteem estudo

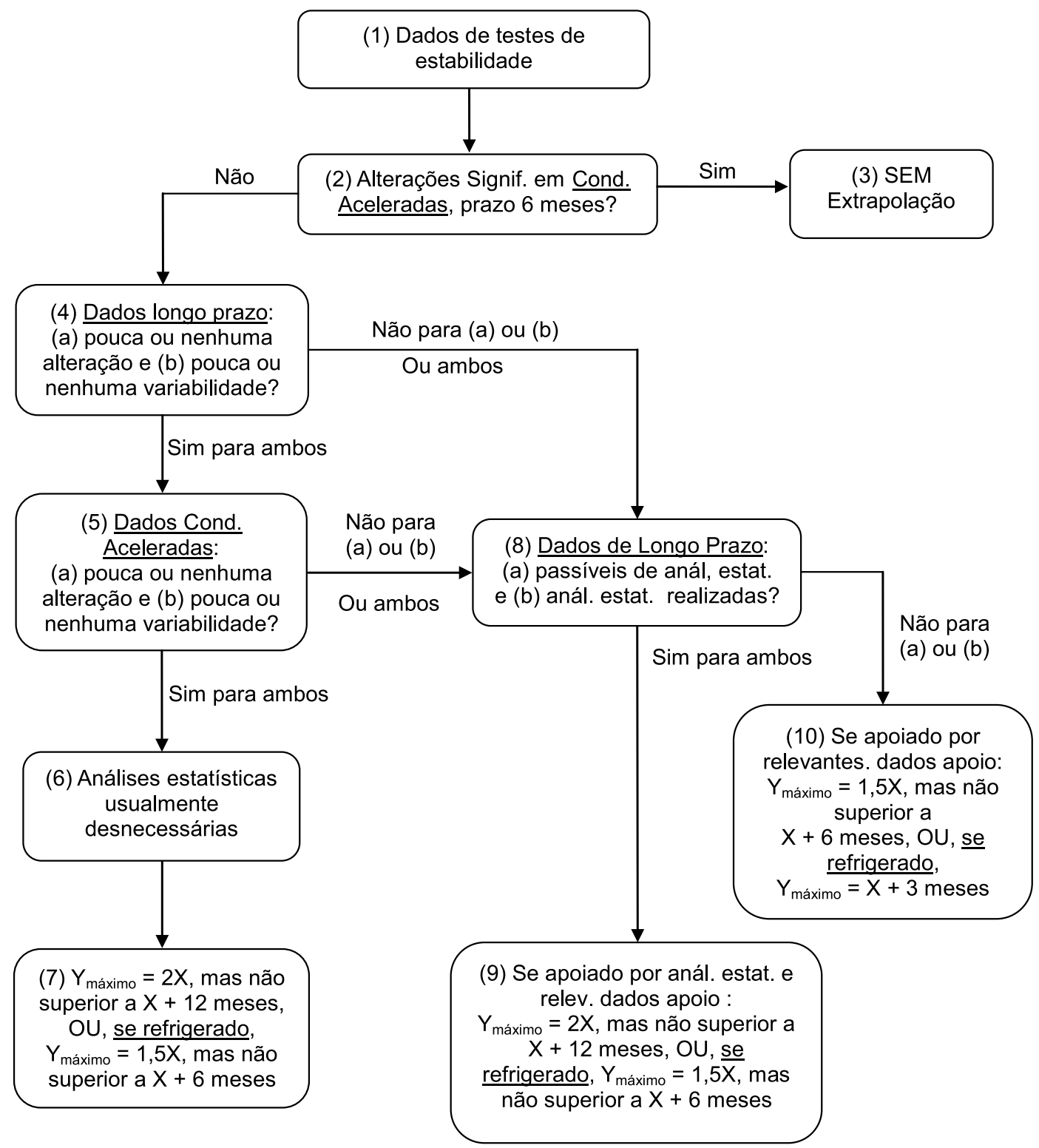

Fluxograma 1S. Extrapolação de dados de longo prazo para proposição de prazo de reteste. Fonte: ICH, Guia Q1E (2003)

*e-mail: mori@feq.unicamp.br 